\title{
Erforschung regionaler Innovationscluster und Aufbau des Forschungsverbundes Regionale Innovationssysteme (RIS)
}

\author{
Martina Brandt, Ulla Große, Frank Hartmann, Rainer Voß
}

\section{Hintergrund des Vorhabens}

Mit der Tendenz zur Globalisierung wirtschaftlicher und gesellschaftlicher Entwicklungsprozesse prägt sich gleichzeitig die Tendenz zu deren Regionalisierung aus. Die Wettbewerbsfähigkeit regionaler Entwicklungsbedingungen in den Bereichen Wirtschaft, Wissenschaft und Bildung, Natur und Umwelt sowie Infrastruktur unterschiedlich dimensionierter Regionen wird immer entscheidender für die Wettbewerbsfähigkeit von Unternehmen, Branchen, Volkswirtschaften, Nationen und Staatenverbünden. Dabei geht es vor allem um den Ausbau regionaler Stärken und die Überwindung regionaler Defizite sowie den Abbau regionaler Disparitäten mit dem Ziel der Erhöhung der Wirtschaftskraft und Lebensqualität in den Regionen. Auf regionaler Ebene verbreitet sich zunehmend die Einsicht, dass eine nachhaltige Wettbewerbsfähigkeit entscheidend durch die Entwicklung und Verknüpfung neuer Technologien und deren Umsetzung in innovative Produkte, Verfahren und Dienstleistungen bestimmt wird.

In Deutschland hat sich in den vergangenen Jahren der Wettbewerb der Regionen, zwischen den Bundesländern, aber auch innerhalb dieser wesentlich verschärft. Im Mittelpunkt stehen dabei z. B. der Aufbau attraktiver und zukunftsfähiger Wissenschafts- und Technologiestandorte, die Ansiedlung, Neugründung und Bestandspflege von Unternehmen, vor allem auch von technologieorientierten Unternehmen. Regionalorientierte Politiken von Bund und Ländern, bezogen auf Wirtschaft, Technologieentwicklung, Bildung, Infrastruktur usw., haben sich insbesondere in den vergangenen 10 Jahren verstärkt an diesen Regionalisierungstendenzen orientiert. Sie wollen diese nutzen und fördern. Ein Ausdruck dessen ist die „Welle“ von bundesweit ausgeschriebenen regionalen Wettbewerben wie BioRegio, BioProfile, EXIST, InnoRegio, Wachstumskerne und Zukunftsregionen für Gründerinnen.

Anfang der 90er Jahre haben einzelne Bundesländer, u. a. Baden-Württemberg, Schleswig Holstein und unter den neuen Bundesländern relativ frühzeitig das Land Brandenburg, komplexe Technologiekonzepte entwickelt und seitdem fortgeschrieben. Wie diese ist auch das aktuell für Brandenburg erarbeitete Landesinnovationskonzept (LIK) hinsichtlich seiner Ziel- und Schwerpunktsetzungen, Potenzialkonzentrationen und Umsetzungsmaßnahmen stark regional orientiert. Es setzt z. B. dort an, wo sich an bestimmten Standorten in den vergangenen Jahren Einrichtungen mit wettbewerbsfähigem wissenschaftlichen und technologischen Know-how entwik- kelt haben, berücksichtigt konzentrierte Standorte mit neugegründeten Unternehmen ebenso wie Synergien zu vorhandenen Branchen und Wirtschaftsstandorten. Die Maßnahmen orientieren auf die Organisation und Stärkung von Potenzialen in bestimmten Teilregionen des Landes, bezogen auf kombinierbare Technologieplattformen, wie z. B. Ernährungstechnologien (in Golm, Rehbrücke) oder Biohybridtechnologien (Golm, Luckenwalde, Rehbrücke, Teltow).

Ein Blick auf die Technologielandkarte des Landes Brandenburg lässt unschwer verschiedene gleichzeitig verfolgte Strategien regionaler Forschungs- und Technologiepolitik erkennen. Einerseits wird versucht, durch eine räumlich möglichst ausgewogene Standortverteilung von Technologiezentren und Hochschulen eine vergleichbare Wirksamkeit in Teilregionen zu erreichen. Andererseits werden ganz gezielt die räumliche Nähe verschiedener Potenziale und ihre möglichen Synergien genutzt, um spezifische konzentrierte Technologiestandorte zu entwickeln (Luckenwalde, Hennigsdorf, Hermannswerder). Dabei wird auch bewusst Redundanz erzeugt, was zusätzliche Wettbewerbsimpulse schafft.

Auch in den Bereichen Arbeitsmarkt- und Wirtschaftspolitik verstärken sich gegenwärtig Regionalisierungstendenzen. Die Durchfuihrung eines Modellvorhabens zur regionalen Existenzgründerförderung im Jahre 2000 (sechs REGO-Modellprojekte) im Land Brandenburg war ein erfolgreicher Versuch, die Wettbewerbsfähigkeit von Regionen in diesem Bereich zu stimulieren.

Bei den Regionalisierungsbestrebungen und der Vielzahl ausgeschriebener regionaler Wettbewerbe handelt es sich offensichtlich weder um einen Modetrend noch um Kampagnen. Diese Aktivitäten treffen vielmehr auf relevante Problemsituationen sowie Interessenschnittflächen der regionalen Akteure aus Wirtschaft, Wissenschaft, Bildung, Politik und Verwaltung. Anders ist nicht zu erklären, dass sich bereits 1995 in Deutschland 17 Regionen als Biotechnologieregionen organisieren konnten und sich im Jahre 1999 weit über 400 Regionen in den neuen Bundesländern an der ersten Phase des InnoRegio-Wettbewerbs beteiligten. In diesen Prozessen wurden die Akteure für solche Wettbewerbsvorteile sensibilisiert, die nur durch regionale Vernetzung zu erreichen sind. Insbesondere für kleine und mittlere Unternehmen, aber auch für Forschungseinrichtungen und Hochschulen sind regionale innovationsorientierte Initiativen eine wirksame Form, um Impulse für die Organisation ihres Ressourcenaustausches zu geben. 
Das zunehmend regional organisierte Austragen und Gestalten von Wettbewerben stellt neue Anforderungen an das strategische Management in Unternehmen, Wissenschafts- und Bildungseinrichtungen und Politik. Traditionelles branchen-, disziplin- und ressortorientiertes Denken und Handeln werden diesen Anforderungen nicht mehr gerecht. Ähnlich wie Unternehmen stehen heute Regionen ebenfalls vor der Aufgabe, differenzierende und fokussierende Wettbewerbsstrategien, vor allem in den Bereichen Forschung, Technologie und Wirtschaft, zu entwickeln.

Eine wesentliche Ursache für die Notwendigkeit von regionaler Differenzierung und Spezialisierung, neben den unterschiedlichen Stärken und Schwächen der Regionen, besteht darin, dass nahezu alle Regionen ihre Hoffnungen in die Potenziale der gleichen zukunftsfähigen komplexen Technologiefelder wie Biotechnologie und IuK-Technologien setzen, um ihre Strukturprobleme zu lösen und ihre Entwicklungsziele zu erreichen. Das Land Brandenburg erklärt mit seinem Landesinnovationskonzept solche Technologieplattformen zu Schwerpunkten, die ein hohes Synergiepotenzial zu anderen Technologien, zu Wirtschaftsbranchen und zu in der Region vorhandenen Potenzialen in Forschung und Wirtschaft aufweisen. Die Aufgabe besteht nunmehr darin, im Spannungsfeld von Bestandspflege/ -entwicklung, Modernisierung, Ansiedlung und Neuimplementation (von Branchen, Standorten, Technologien) geeignete Organisationsformen, Instrumentarien und Fördermaßnahmen zur Umsetzung dieses Konzepts zu finden. Gleichzeitig muss rechtzeitig durch Management und Politik gegengesteuert werden, damit sich nicht durch schwerpunktorientierte regionale Technologieentwicklung neue regionale Disparitäten aufbauen.

Die Organisation und Entwicklung Regionaler Innovationssysteme verschiedenen Typs und unterschiedlicher Spezifikation wird als eine moderne und zukunftsfähige Strategie zur Erhöhung regionaler Wettbewerbsfähigkeit gesehen. Weltweit wurden insbesondere in der zurückliegenden Dekade die Forschungen hierzu intensiviert [1].

Regionale Innovationssysteme werden, bezogen auf unterschiedliche Regionen, Wirtschaftsbereiche und Technologiefelder, insbesondere unter folgenden Gesichtspunkten untersucht:

- Ursachen, Bedingungen und Voraussetzungen

- Ziele und Funktionen

- Typen, Organisationsformen

- Elemente, Potenziale und Strukturen, Proportionalität

- Entstehungs- und Entwicklungsprozesse

- Kriterien fuir Ausprägung und Reife

- Zusammenhänge und Übergänge zwischen Typen

- Interessen und Verhalten der Akteure

- Spezifika von und Kooperation zwischen regionalen Innovationssystemen

- Wirkungen/Folgen von regionalen Innovationssystemen und ihrer Elemente
- Stärken und Schwächen sowie Vor- und Nachteile von Typen regionaler Innovationssysteme

- Management und Politik

- Instrumente und Methoden

Die Untersuchungen erfolgen durch verschiedene sozial-, wirtschafts- und regionalwissenschaftliche sowie psychologische Disziplinen. Insbesondere in der regionalorientierten Innovationsforschung wird international und national versucht, diese Disziplinen themen- bzw. projektbezogen zusammenzuführen, ohne deren Selbständigkeit aufzuheben. Im Land Brandenburg gibt es bisher kein institutionalisiertes Forschungspotenzial, das diese Aufgaben bewältigen könnte.

\section{Zielsetzung}

Basierend auf den Erfahrungen und Ergebnissen der 5-jährigen engen Kooperation zwischen der Technischen Fachhochschule Wildau und dem Institut für Regionale Innovationsforschung wird das Ziel verfolgt, zunächst ausgehend von Potenzialen des Landes Brandenburg, einen Forschungsverbund zu regionalen Innovationssystemen (RIS) aufzubauen [vgl. Abbildung 1]. In diesem Forschungsverbund sollen insbesondere die überwiegend an den Hochschulen des Landes arbeitenden wirtschafts-, sozial- und humanwissenschaftlichen Forschungspotenziale, aber auch außeruniversitäre Einrichtungen eingebunden und zu multi- bzw. interdisziplinärer Arbeit angeregt werden. Darüber hinaus gibt es in einigen naturwissenschaftlichen Forschungseinrichtungen des Landes Gruppen, die sich mit verwandten Themen beschäftigen und zur Mitarbeit eingeladen werden. Schließlich können die Erfahrungen solcher Akteure in die Erforschung Regionaler Innovationssysteme eingebracht werden, die im Rahmen des InnoRegio-Wettbewerbs konzeptionell gearbeitet haben.

Der Forschungsverbund zu Regionalen Innovationssystemen hat folgende Hauptfunktionen:

- Aufbau und Vertiefung der Kooperation zwischen Hochschulforschung und außeruniversitärer Forschung

- Herausarbeitung wissenschaftlich attraktiver und praxisorientierter Forschungsthemen

- Entwicklung interdisziplinärer Verbundprojekte und Erhöhung der Drittmittelfähigkeit sowie Erschließung neuer Finanzierungsquellen auf Landes-, Bundes- und EU-Ebene

- Aufbau ausgewählter internationaler Kooperationsbeziehungen

- Entwicklung ganzheitlich orientierter Instrumente und Methoden für Management und Politik von regionalen Innovationssystemen und Unterstuitzung der Anwendung durch Akteure in Wirtschaft, Wissenschaft, Politik und Verwaltung

- Breite Umsetzung der Forschungsergebnisse in Lehrund Ausbildungsinhalte

- Erhöhung der Öffentlichkeitswirksamkeit der Forschungsergebnisse durch zusätzliche Multiplikationseffekte 
Der Forschungsverbund soll Orientierungs- und Handlungswissen sowie Instrumente und Methoden als Lösungsbeiträge für folgende Fragestellungen zur Verfügung stellen:

1. Wo liegen Ansatzpunkte im Land Brandenburg, um mit Strategien in den Bereichen Wissenschafts-, Bildungs-, Technologie-, Wirtschafts- und Arbeitsmarktpolitik verstärkt zur Entwicklung regionaler Innovationssysteme beizutragen und diese fuir die Realisierung entsprechender Entwicklungsziele zu nutzen?

2. Welche bisherigen Fördermaßnahmen können regionale Innovationssysteme besonders unterstuitzen? Wie sollten sie modifiziert, ggf. kombiniert werden? Ergeben sich aus der Profilierung von regionalen Innovationssystemen Ansätze für die Ausrichtung von Förderschwerpunkten des Landes?

3. Welche Konsequenzen hätte eine stärkere Orientierung auf regionale Innovationssysteme für die Regional- und Standortpolitik des Landes? (Entwicklung von Agglomerationen, peripheren Regionen, Technologiezentren als Kristallisationskerne für Cluster...)

4. Welche Akteure und Institutionen in den Regionen haben, bezogen auf Innovationscluster, welche Funktionen und wie müssen diese inhaltlich und organisatorisch ausgerichtet sein (z. B. neue Formen des Technologietransfers)? Welche Funktionen und Akteure fehlen und können wie von außen verfügbar gemacht werden (z. B. Akquisitions- und Ansiedlungsstrategien, Kooperation mit anderen Regionen)?

5. Wie kann man die Attraktivität und Wettbewerbsfähigkeit regionaler Innovationssysteme messen und vergleichen?

6. Welche Anforderungen ergeben sich an innovative Finanzierungsmodelle unter dem Gesichtspunkt regionaler Innovationscluster?

7. Wie lassen sich durch Clustermanagement die Risiken komplexer Innovationsprozesse mindern und die Chancen erhöhen?

8. Können langfristige Szenarien über die Entwicklung von Technologien und Märkten für zukunftsfähige Innovationscluster zur Begründung von Bildungsund Ausbildungsprofilen genutzt werden?

9. Welche regionalen Muster der Clusterbildung sind im Vergleich mit anderen Regionen wie wettbewerbsfähig? Welche Konsequenzen hat das für eine Clusterpolitik?

10. Wie kann die Etablierung und Entwicklung regionaler Innovationscluster regional und überregional beeinflusst werden?
Der Aufbau einer derartigen neuen Forschungsstruktur in Form eines Verbundes setzt neben der Bereitschaft zur Mitwirkung erfahrungsgemäß voraus, dass es gelingt, einen durch entsprechende Kompetenzen getragenen „Strukturknoten“ zu profilieren,

- der Impulse für die interdisziplinäre Arbeitsweise des Verbundes gibt,

- der Kooperationsbeziehungen aufbaut und

- der aufgrund eigener Arbeiten immer wieder dazu anregt, die erzielten Ergebnisse in Dienstleistungsangebote für das Management regionaler Innovationssysteme umzusetzen.

Zu diesem Zweck wird gegenwärtig an der TFH Wildau eine Forschungsgruppe „Regionale Innovationscluster" aufgebaut, die selbst Forschungsprojekte bearbeitet und akquiriert und Koordinierungsfunktionen für den Verbund entwickelt und in seiner Aufbauphase wahrnimmt. Diese Forschungsgruppe wird von Herrn Professor Voß geleitet, ebenso wie das Institut für Regionale Innovationsforschung, das seit mehreren Jahren gemeinsam mit der TFH Wildau Forschungsprojekte zu ausgewählten Fragestellungen regionaler Innovationssysteme bearbeitet und künftig auch eng mit der Forschungsgruppe kooperieren wird.

\section{Arbeitsschwerpunkte für den Forschungs- verbund Regionale Innovationssysteme und die Forschungsgruppe Regionale Innovationscluster}

Es werden zunächst folgende inhaltliche Schwerpunkte der Forschung und daraus abzuleitende Dienstleistungsaktivitäten und Inhalte für die Lehre gebildet, die durch weitere Verbundteilnehmer modifiziert und erweitert werden können.

\subsection{Zukunftsfähige Organisationsformen regionaler Innovationssysteme}

Die zunehmende Globalisierung der Märkte, die Beschleunigung der Technologieentwicklung und die Intensivierung des Wettbewerbs zwingen Unternehmen dazu, neue und effizientere Organisationsformen zur Hervorbringung, Herstellung und Vermarktung von Produkten und Dienstleistungen zu entwickeln. Klassische Formen der vertikalen Integration sowie die bisherige Branchenorientierung stoßen an Grenzen der weiteren Erhöhung der Innovativität und Produktivität. Als besonders geeignete Organisationsformen werden weltweit Innovationsnetzwerke, innovative Milieus und in jüngster Zeit vor allem Innovationscluster erforscht, entwickelt und genutzt, um auf die zunehmenden Unsicherheiten zu reagieren, die durch die wachsende Komplexität und Dynamik in den Entwicklungen von Technologien, Märkten und Rahmenbedingungen entstehen.

Netzwerke [2] stellen eine unter dynamisierten Umweltbedingungen besonders geeignete und innovationsbegünstigende Organisationsform der Wahrnehmung, 


\section{Forschungsverbund Regionale Innovationssysteme (RIS)}

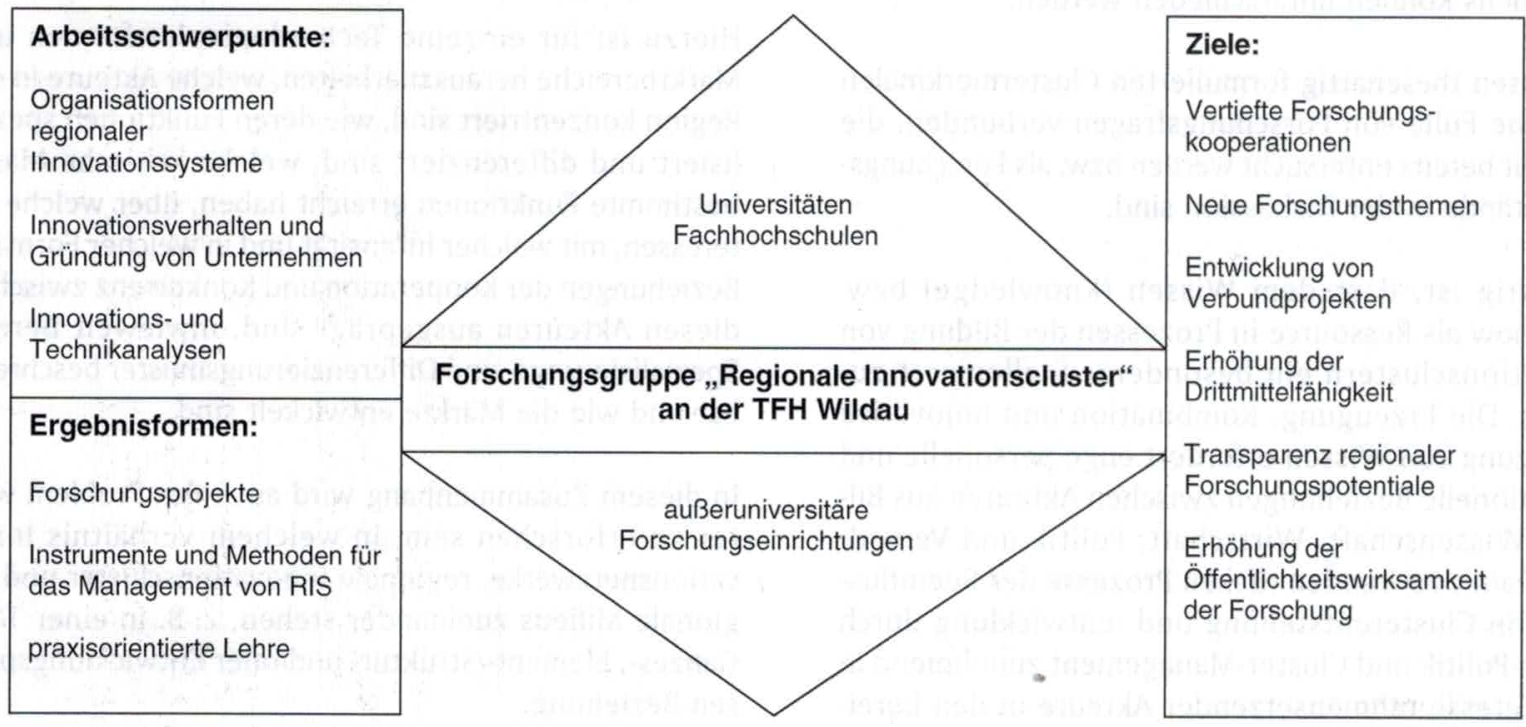

Abb. 1: Forschungsverbund RIS im Überblick

Gestaltung, Koordination und Beeinflussung des innovationsrelevanten Verhaltens von Akteuren innerhalb und außerhalb des Netzes dar. Netzwerkbeziehungen werden dabei verstanden als über vertraglich geregelte und damit relativ dauerhafte kooperative Beziehungen hinausgehende Akteursbeziehungen zum Zwecke der Hervorbringung und Durchsetzung von Innovationen. Solche Innovationsnetzwerke [3] erfüllen drei Hauptfunktionen:

1. Die Reduzierung von technologischen Unsicherheiten hinsichtlich der sachlichen und zeitlichen Machbarkeit. Dabei werden bereits frühe Anwendungsinteressen und dadurch vermittelt marktrelevante Orientierungen einbezogen.

2. Die Reduzierung von Marktunsicherheiten durch das Schaffen von Anwendungsbeispielen (Prototypen) und die Beeinflussung des Marktbildungsprozesses (Pilotkunden). Diese marktbildenden Aktivitäten werden wiederum häufig auf die Aktivitäten im Prozess der Technikentwicklung zurüickgekoppelt. Es gibt noch einen „point of return“ ohne kumulierte Ressourcen- und Investitionsverluste.

3. Die Reduzierung von Planungsunsicherheiten im Hinblick auf Technikentwicklung ebenso wie auf Marktbildungsprozesse.

Regionale Cluster bringen auf neue Art und Weise Technologien, Branchen und Anwendungsbereiche in Zusammenhang und erhöhen die Produktivität und Wettbewerbsfähigkeit der beteiligten Unternehmen und der Regionen gleichermaßen [4].

Folgende generelle Merkmale von Clustern können hervorgehoben werden:
1. Ausrichtung auf etablierte, entwicklungsfähige und heterogene Endmärkte. Gleichzeitig gibt es hochdifferenzierte Marktsituationen innerhalb des regionalen Clusters.

2. Neben Unternehmen mit technisch-technologischen Kernfunktionen im Cluster spielen Wissenschafts- und Forschungseinrichtungen, spezifische Zulieferer, produktionsnahe Dienstleister, Vertriebsorganisationen sowie regulierende und rahmensetzende Akteure eine Rolle.

3. Gleichzeitigkeit von flexibler Spezialisierung und darauf bezogener funktionaler Differenzierung.

4. Keine Einzigartigkeit, aber typische Ausprägung. Das Typische wird durch die jeweiligen Spezialisierungsund Differenzierungsmuster der Cluster erzeugt. Die dadurch entstehende Exklusivität des räumlich konzentrierten Know-hows führt zur Grenze der Imitierbarkeit durch andere Regionen.

5. Wichtigkeit einer kritischen Masse. Die erforderlichen Funktionen müssen mehrfach in der Region in einer bestimmten Spezialisierung und Differenzierung durch kompetente Akteure angeboten werden. Dadurch wird eine produktive Verbindung von Wettbewerb und Kooperation möglich und die Muster der Beziehungen zwischen den regionalen Akteuren können bei sich wandelnden Anforderungen der Märkte oder des technologischen Know-hows wechseln.

6. Endogene Kernpotenziale als Basis der Clusterbildung. Akquisition und Ansiedlung fehlender Funktionen können das Cluster stärken oder vervollständigen. Impulse von außen können dabei als Katalysatoren wirken. 
7. Längere Entstehungs- und Entwicklungsgeschichte. Phasen der Entstehung, des Wachstums, der Restrukturierung und Erneuerung bzw. des Alterns und Absterbens können unterschieden werden.

Mit diesen thesenartig formulierten Clustermerkmalen sind eine Fülle von Forschungsfragen verbunden, die weltweit bereits untersucht werden bzw. als Forschungsgegenstände in der Diskussion sind.

Unstrittig ist, dass dem Wissen (Knowledge) bzw. Know-how als Ressource in Prozessen der Bildung von Innovationsclustern ein besonderer Stellenwert zukommt. Die Erzeugung, Kombination und innovative Umsetzung von Wissen erfordert enge personelle und institutionelle Beziehungen zwischen Akteuren aus Bildung, Wissenschaft, Wirtschaft, Politik und Verwaltung. Damit verbunden rüicken Prozesse der Beeinflussung von Clusterentstehung und -entwicklung durch Cluster-Politik und Cluster-Management zunehmend in das Interesse rahmensetzender Akteure in den Bereichen Forschungs-, Technologie- und Wirtschaftspolitik. Dièse können Clusterbildungsprozesse z. B. durch geeignete Fördermaßnahmen in clusterrelevanten Technologiebereichen, den Aufbau administrativer Bereiche bezogen auf Cluster und die Schaffung innovationsfördernder Standards auf der Nachfragerseite unterstiutzen.

Im Rahmen des Forschungsverbundes soll für verschiedene Technologieplattformen und Marktbereiche, besonders auch des Landesinnovationskonzeptes, untersucht werden, welche Anzeichen und Bedingungen es für Clusterbildungsprozesse gibt, welche Rolle kleine und mittlere Unternehmen dabei spielen und wie durch Clusterpolitik solche Prozesse unterstuitzt werden können.

Dies impliziert u. a. folgende Aufgaben:

1. Weiterentwicklung des Konzepts „Regionale Innovationscluster" und dessen Operationalisierung für ausgewählte Anwendungsbereiche.

2. Beobachtung von Entwicklungen in relevanten Bereichen in Wissenschaft und Wirtschaft (Trends, regionale Konzentrationen von Potenzial, Differenzierungspotenziale und Spezialisierungen, Wettbewerbssituationen, Netzwerkbildungen) und Identifizierung von Ansätzen für Clusterbildung (z. B. Unternehmen als Nukleus bzw. Kompetenzzentrum als Kristallisationskern).

3. Analyse und Bewertung ausgewählter Brandenburger Regionen. Dabei stehen solche Regionen im Mittelpunkt, die sich noch im InnoRegio-Wettbewerb befinden, die sich an Vorphasen des Wettbewerbs beteiligt hatten und mit ihren Zielen und Potenzialen im Zusammenhang mit Technologieplattformen und Marktfeldern des Landesinnovationskonzeptes stehen sowie diejenigen, die sich am neuen Wettbewerb „Wachstumskerne" beteiligen.
4. Kontinuierliche Ableitung von Schlussfolgerungen aus der Analyse und Begleitung fuir die Clusterpolitik und das Clustermanagement in der Region.

Hierzu ist für einzelne Technologieplattformen und Marktbereiche herauszuarbeiten, welche Akteure in der Region konzentriert sind, wie deren Funktionen spezialisiert und differenziert sind, welche kritische Masse bestimmte Funktionen erreicht haben, über welche Interessen, mit welcher Intensität und in welcher Form die Beziehungen der Kooperation und Konkurrenz zwischen diesen Akteuren ausgeprägt sind, inwieweit bereits Spezialisierungs- und Differenzierungsmuster beschreibbar und wie die Märkte entwickelt sind.

In diesem Zusammenhang wird auch das Problem weiter zu erforschen sein, in welchem Verhältnis Innovationsnetzwerke, regionale Innovationscluster und regionale Milieus zueinander stehen, z. B. in einer Teil-/ Ganzes-, Element-/Struktur- und/oder Entwicklungsphasen-Beziehung.

Die Forschungsgruppe „Regionale Innovationscluster“ beabsichtigt hierzu die Bearbeitung folgender Forschungsvorhaben:

- Rolle unterschiedlicher Lernprozesse für die Wissenstransformation in regionalen Innovationssystemen unter besonderer Beachtung von tacit knowledge

- Bewertung der Entwicklungspotenzen ausgewählter Brandenburger Innovationsregionen in Bezug auf eigenständige Clusterbildungen oder als Komponenten von Clustern sowie Konsequenzen für Clustermanagement

- Biotechnologiebasierte Clusterbildung in der Region Berlin-Brandenburg

- Modellentwicklung für Cluster „Seniorengerechte Technik" in Berlin-Brandenburg

- Ziele, Strukturen und Wirkungen des „Brandenburger Netzwerks Alternative Energietechnologien“"

- Indikatoren zur Messung und Bewertung regionaler Innovationssysteme

Die Mitarbeiter der Forschungsgruppe verfügen ebenso wie das mit ihr kooperierende Institut für Regionale Innovationsforschung über langjährige Erfahrungen insbesondere mit Analysen und Forschungsprojekten zum Zusammenhang von Biotechnologie und Regionalentwicklung. Untersuchungsgegenstände waren ganze „Biotechnologielandschaften “(Ebene Bundesland), Teilregionen und konzentrierte Technologiestandorte [5]. Das Technologiefeld „Seniorengerechte Technik“ wird ebenfalls seit einigen Jahren untersucht, wobei der Schwerpunkt in der Region Südbrandenburg lag [6].

\subsection{Innovationsverhalten und Gründung von Unter- nehmen in regionalen Innovationssystemen}

Forschende, produzierende und dienstleistende innovative Unternehmen bilden den Kern regionaler Innovationssysteme, ohne immer auch gleichzeitig der Ausgangspunkt für deren Bildung zu sein. Letztlich muss jede Region über eine kritische Masse an innovativen 
Unternehmen verfügen, um die Lösungspotenziale von Technologien durch neue Produkte, Verfahren und Dienstleistungen umsetzen zu können. Damit rückt das Innovationsverhalten bereits in der Region vorhandener bzw, neu zu gründender bzw. anzusiedelnder Unternehmen in den Mittelpunkt von Aktivitäten zur Organisation von regionalen Innovationssystemen [7].

Das Innovationsverhalten von Unternehmen sollte auch regional orientiert werden, d. h. regionale Ressourcen und regionale Nähe sind zu nutzen (Vernetzung), um zur Lösung regionaler Entwicklungsprobleme beizutragen. Damit sind vielfältige Forschungsfragen mit Relevanz für das Innovationsmanagement in Unternehmen und für das Management bezogen auf die Politik verbunden. Diese betreffen

- das Verhältnis von kleineren und größeren Unternehmen in regionalen Innovationssystemen und deren jeweiliges Innovationsverhalten,

- Probleme der Wahrnehmung, Bewertung und des Umgangs von Unternehmen mit ihren Umfeldakteuren,

- die Beziehungen zwischen TOU, Zulieferern und wirtschaftsnahen Dienstleistern,

- den Wissens- und Technologietransfer von Forschungseinrichtungen in Firmen,

- neue Ansätze und Möglichkeiten zur Unterstützung von Unternehmen in Vor-, Gründungs- und Stabilisierungsphasen,

- die Unterstützung von Firmen hinsichtlich Frühphasen im Technologiemanagement (scanning, monitoring) und Marketing (advanced marketing...).

Die TFH Wildau und ihr langjähriger Kooperationspartner, das Institut für Regionale Innovationsforschung, verfuigen über ausgewiesene Kompetenzen auf dem Gebiet der mittelstands- und technologieorientierten Innovationsforschung. In einer Vielzahl von Projekten wurde zu den Themen „Innovationsprozesse in und Unternehmensanforderungen an KMU“, „Marktpotenziale für innovative Produkte und Verfahren von KMU“, „Konzentrierte Technologiestandorte bzw. technologiebasierte KMU und nachhaltige Entwicklung von Regionen“, „Qualifizierungsbedarfe von KMU“, „Internationale Technologietrends mit Relevanz für KMU“ gearbeitet. Dabei wurde unter verschiedenen Sichten untersucht, wie das Innovationsverhalten von Unternehmen im Interesse der Lösung regionaler Entwicklungsprobleme genutzt und gestaltet werden kann. Die Untersuchungen bezogen sich neben Berlin-Brandenburg auf Mecklenburg-Vorpommern, Nordrhein-Westfalen, Sachsen-Anhalt und Sachsen.

Es konnte gezeigt werden, dass für den unternehmerischen Innovationsprozess (und dessen regionale Ausstrahlung) nicht in erster Linie das Vorhandensein/Nichtvorhandensein oder die qualitative Ausprägung interner und externer Einflussfaktoren, sondern der Umgang mit diesen Faktoren von Bedeutung ist. Dieser Umgang kann in der Wahrnehmung, Bewertung und dem Handeln bestehen. Dabei wiederum nimmt nicht nur das Innovationsverhalten der Unternehmen selbst, sondern auch das Verhalten von Umfeldakteuren Einfluss [8].
Das genutzte faktorbasierte, gleichzeitig aber auch verhaltensorientierte Forschungskonzept zielt darauf ab, Innovationsverhalten auf Technologiefeldern zu beschreiben, zu erklären und Gestaltungsansätze für dessen verstärkte regionale Orientierung und Wirksamkeit aufzuzeigen. Fokussiert auf das innovative Verhalten der Unternehmen im Prozess der Kombination externer und interner Ressourcen geht das Konzept davon aus, dass durch intensive Ressourcenaustauschprozesse Einflussmöglichkeiten und Ansatzpunkte für eine regionale Orientierung und Wirksamkeit des Innovationsverhaltens gegeben sind. Im Zusammenhang mit der Analyse des Einflusses regionaler Umfelder ist hier auch das vernetzte Handeln von Akteuren bedeutsam.

Zum Zusammenhang der nachhaltigen Entwicklung von Regionen mit technisch-technologischen Innovationen liegen ebenfalls Ergebnisse aus Projekten an der Schnittstelle zwischen unternehmensbezogener und lokaler Nachhaltigkeitsbetrachtung vor [9]. Zum einen werden aufgrund der territorialen Verankerung wirtschaftlicher Aktivitäten, der Schaffung attraktiver, kulturell eingebetteter Milieus und der Herausarbeitung unverwechselbarer Identitäten und Entwicklungsleitbilder Regionen auch bei der Umsetzung der Forderungen aus der Agenda 21 eine herausragende Rolle spielen [10]. Zum anderen reichen Nachhaltigkeitskriterien, die auf das Unternehmen an sich (also als Einzelelement) bezogen sind [11] nicht aus, um die Rolle der Wirtschaft für nachhaltige regionale oder kommunale Entwicklungsprozesse zu beschreiben. Sie stellen vielmehr nur eine notwendige, aber nicht hinreichende Voraussetzung hierfür dar. Daher wurde das Konzept eines die Region charakterisierenden Systems regionaler Entwicklungsbedingungen zugrunde gelegt, in dem die Wirtschaft eine wichtige und mit anderen Entwicklungsbedingungen stark vernetzte Bedingung ist. Wiederum sind Akteure innerhalb und außerhalb der Unternehmen durch ihren Umgang mit den regionalen Entwicklungsbedingungen an der Realisierung einer nachhaltigen Entwicklung beteiligt. Auf der Grundlage des o. g. Konzeptes erfolgte im Jahr 2000 auch die wissenschaftliche Evaluation der sechs Modellprojekte der „Regionalen Existenzgründungsoffensiven" des Landes Brandenburg, welche die Existenzgründer durch Investitionen in ihr gründungsrelevantes Know-how unterstiitzten [12].

Anknüpfend an diese Ergebnisse könnten im Rahmen des Verbundes folgende Themenstellungen vertieft untersucht werden:

- Regionale Orientierung des Innovationsverhaltens von KMU durch Transformation regionaler Problemlagen in Märkte

- Zusammenhang von Innovationsverhalten und regionaler Technologiediffusion

- Determinanten der Herausbildung von Innovationsverhaltensmustern und deren langfristiger Stabilität und Erneuerung

- Einfluss von Veränderungen in Innovationsbedingungen auf das Verhalten von Unternehmen und das innovationsorientierte Verhalten von Akteuren in Unternehmensumfeldern (Absatz- und Beschaffungs- 
märkte, Wissenschaft und Technologie, gesellschaftliche Rahmenbedingungen)

- Messung des Innovationsverhaltens mittelständischer Unternehmen

Die Forschungsgruppe beabsichtigt hierzu die Bearbeitung folgender Vorhaben:

- Konzipierung eines „Demonstrationszentrums Biotechnologie“ in der Stadt Luckenwalde

- Wirkungsanalyse zu den Brandenburger REGOs: Gründungsqualität, Bestandsfestigkeit, Beiträge von geförderten/gecoachten Unternehmen zur Stärkung der Regionalwirtschaften

- Gender Mainstreaming in der Gründerförderung

- Begleitung und Aufbau eines Kompetenzzentrums Existenzgründerförderung

- Beiträge zur Weiterentwicklung des Curriculums Unternehmensgründung an der TFH Wildau

- Beiträge Brandenburger Fachhochschulen für die wettbewerbsfähige Entwicklung und Anwendung von Technologien in mittelständischen KMU

- Methodenentwicklung zur Abschätzung regionaler, dynamischer Marktpotenziale für radikale Innovationen von KMU

\subsection{Innovations- und Technikanalysen für ausgewählte Technologieplattformen und innovative Wirtschaftsbereiche}

Unter Innovations- und Technikanalysen (ITA) werden in jüngster Zeit externe Dienstleistungen insbesondere für Wirtschaftssubjekte (Unternehmen, Verbände) verstanden, die über „klassische“ Technikfolgenabschätzung (bisher konzeptionell überwiegend auf die Beratung des politischadministrativen Systems ausgerichtet) hinausgehen [13]. Auch im Rahmen der Erforschung Regionaler Innovationssysteme sollen Innovations- und Technikanalysen für die beiden Zielgruppen „Politisch-administratives System“ und „Wirtschaft" durchgeführt werden. Ergebnisformen solcher Innovations- und Technikanalysen sind z. B. Studien zu Folgewirkungen des Technikeinsatzes, strategische Szenarien zur Ableitung von Handlungsoptionen, Bedarfs- und Nachfrageanalysen/Anforderungsprofile, Technikpotenzialanalysen, Technikakzeptanzstudien, Studien zur Technikbeurteilung, Technologiefrüherkennungsstudien (Experten-Delphis, Trendforschungen), Forecasting-Prozesse, Konfliktbearbeitungsdienste, aber auch Beratungs- und Kommunikationsunterstiitzung.

Die TFH Wildau und das IRI verfügen über ausgewiesene konzeptionelle, methodische und empirische Kernkompetenzen auf dem Gebiet der ITA und haben in den vergangenen Jahren sowie gegenwärtig im Rahmen der Akquisition von Projekten des Bundesministeriums für Bildung und Forschung (BMBF) gezeigt, wie Kooperation zur Erhöhung der Kompetenz, Kapazität und Drittmittelfähigkeit fuihren kann. Dies basiert nicht zuletzt auf den Erfahrungen, die beide Partner in einem antizipativen, innovationslinienorientierten TA-Verfahren im Bereich der Pflanzengentechnik (gefördert durch die Ministerien für Umwelt und Wissenschaft des Landes Brandenburg) gesammelt haben [14]. Zwei ITA-Projekte des
BMBF zur Problematik „Akzeptanz und Nachfragemuster als Standortvorteile“ (Laufzeit November 2000 bis April 2002) werden auf den Technologiegebieten „Pflanzengentechnik“ und „Seniorengerechte Technik“" arbeitsteilig zwischen der TFH und dem IRI bearbeitet. Sie bilden in der Startphase den inhaltlichen „Kern“ der Arbeiten der im Aufbau befindlichen Forschungsgruppe „Regionale Innovationscluster".

Weitere Arbeitsschwerpunkte der Forschungsgruppe sind:

- Innovations- und Technikanalysen im Bereich Life Sciences und Biotechnologie

- Bedarfsanalysen der Wirtschaft nach externen ITADienstleistungen

- Konzepte und Methoden für Innovations- und Technikanalysen

- Integration von ITA-Fragestellungen in die Forschung und Ausbildung von Ingenieuren und Betriebswirten an Fachhochschulen

Für die gemeinsame Weiterentwicklung solcher Themenfelder mit Partnern bringt der Leiter der Forschungsgruppe seine Erfahrungen und Kontakte als Vorsitzender des Beirates des Biotechnologieverbundes BerlinBrandenburg und als Sprecher des wissenschaftlichen Beirates des Biotechnologieparkes Luckenwalde ein. Er verfügt über enge Beziehungen zu den Biotechnologiezentren der Region, zu Forschungseinrichtungen und Unternehmen sowie Akteuren aus Politik und Verwaltung. Darüber hinaus arbeitet er im Expertengremium „Innovations- und Technikanalyse" beim BMBF mit und verfügt über Kontakte zu den entsprechenden Forschungseinrichtungen in Deutschland. Da die Strategie des BMBF dahin geht, die Kompetenzen dieser Einrichtungen stärker zu verzahnen und auf Bedarfe auch der Wirtschaft zu orientieren, sind hier Kooperationsmöglichkeiten auszuloten und gezielt aufzubauen. Damit könnte ein möglicher Arbeitsschwerpunkt „Innovationsund Technikanalysen im Rahmen regionaler Innovationssysteme" in die deutsche TA-Szene integriert werden.

\subsection{Konzipierung und Unterstützung des Aufbaus des Forschungsverbundes}

Der Aufbau des Forschungsverbundes RIS ist eine permanente Arbeitsaufgabe, die vom Leiter der Forschungsgruppe und deren Mitarbeitern geleistet werden soll. Dazu gehört neben der wissenschaftlichen Konzipierung und Koordinierung des Verbundes auch die aufwendige Initiierung und Pflege von Kontakten der Verbundteilnehmer sowie die Konzipierung und Umsetzung des Projektmanagements.

Im Juni 2001 wird ein Auftaktworkshop durchgefuihrt und ausgewertet. Inhaltliche Schwerpunkte sind der Interessenabgleich und die Abstimmung des von der Forschungsgruppe vorgeschlagenen Forschungsprofils des Verbundes. Darauf basierend werden in den Folgejahren interdisziplinäre Kooperationsprojekte entwickelt. Dazu werden, je nach Bedarf, weitere Veranstaltungen durchgefuihrt. 
Eine wichtige Aufgabe besteht darin, Quellen für die Finanzierung von Projekten des Forschungsverbundes zu erschließen. Entsprechende Überlegungen sind Bestandteil der Konzipierung des Verbundes im ersten Jahr und sollten sich auf Möglichkeiten zur Bündelung von bzw. Prioritätensetzung bei Ressortfördermitteln des Landes, auf die Einwerbung von Stiftungsmitteln auf Landes- und Bundesebene, von Mitteln der Deutschen Forschungsgemeinschaft sowie Fördermitteln der Bundesministerien und der EU erstrecken. Erfahrungen unterschiedlicher Einrichtungen bei der Drittmittelakquise können eingebracht und deren „Gewicht“ durch Zusammenschluss erhöht werden.

Es ist vorgesehen, einen Beirat des Verbundes zu bilden. Die inhaltliche Koordinierung des Verbundes sollte periodisch alternierend von interessierten Mitgliedseinrichtungen wahrgenommen werden. Durch einen Internetauftritt und andere PR-Maßnahmen soll der Forschungsverbund in der Region und darüber hinaus bekannt gemacht werden.

Die Chancen für den Aufbau des Forschungsverbundes Regionale Innovationssysteme im Land Brandenburg werden aus folgenden Gründen als günstig bewertet:

1. Es handelt sich um ein international attraktives und wettbewerbsrelevantes Forschungsgebiet.

2. Es bestehen auf verschiedenen Landes- und Regionalebenen Problemlösungsbedarfe, die bedient werden können.

3. Es gibt wettbewerbsfähige Kernkompetenzen zur Erforschung und zum Management von Komponenten regionaler Innovationssysteme im Land Brandenburg bzw. in Berlin.

4. Sowohl das Ministerium für Wissenschaft, Forschung und Kultur als auch das Ministerium für Wirtschaft, Mittelstand und Technologie, vertreten durch die T.IN.A., haben ein solches Vorhaben begrüßt.

5. Die Anfinanzierung der Forschungsgruppe als „Strukturknoten" des Forschungsverbundes ist gegeben.

6. Aufgrund der Lage von Brandenburg als Grenzregion der EU und der intensiven Beziehungen der TFH Wildau zu verschiedenen mittel- und osteuropäischen Ländern (z. B. zu Weißrussland, Polen, Tschechien) bestehen günstige Voraussetzungen, die Forschungen zu regionalen Innovationssystemen mit Projekten und Kooperationen zu diesen Ländern zu bereichern.

Der erfolgreiche Aufbau des Forschungsverbundes (Umfang, Tempo, Ausstrahlung) hängt ganz wesentlich davon ab, welche Akteure sich mit welchen Konzepten, Kompetenzen und Leistungsangeboten in den Forschungsverbund einbringen werden und wie dieser durch Politik, Verwaltung und Wirtschaft - insbesondere im Land Brandenburg - wahrgenommen und unterstuitzt wird.

\section{Literatur}

[1] vgl. Porter, M. E.: The competitive advantage of nations, New York 1990. Lundvall, B. A.: Innovation as an interactive process. From user-producer interaction to the national system of innovation. In: Dosi, G., Freeman, C., Nelson, R., Silverberg, G. (Hg.): Technical Innovation and Economic Theory. London 1988, S. 349-369. Nelson, R.: National Innovation Systems. A comparitive Analysis. Oxford, 1993. Porter, M. E.: On Competition, Boston 1998. Braczyk, H-J., Cooke, P., Heidenreich, M. (Hg.): Regional Innovation Systems, London 1998. Fuchs, G., Krauss, G., Wolf H-G.: (Hrsg.) Die Bindungen der Globalisierung. Interorganisationsbeziehungen im regionalen und globalen Wirtschaftsraum. Marburg 1999.

[2] vgl. z. B. Camagni, R. (Hrsg.): Innovation networks: spatial perspectives. London 1991. Fritsch, M.: UnternehmensNetzwerke im Lichte der Institutionenökonomik. In: Jahrbuch für Neue Politische Ökonomie. 11. Band, Tübingen 1992, S. 89-102. Grabher, G.: Lob der Verschwendung. Redundanz in der Regionalentwicklung. Ein sozioökonomisches Plầdoyer. Berlin 1994. Fürst, D. und Kilper, H.: Die Innovationskraft regionaler Politiknetzwerke. Gelsenkirchen o. J.

[3] vgl. Kowol, U.: Innovationsnetzwerke. Technikentwicklung zwischen Nutzungsvisionen und Verwendungspraxis. Wiesbaden 1998

[4] vgl. Porter, M. E.: Clusters and the new economics of competition. In: Harvard Business Review. November December 1998, p. 78

[5] vgl. Voß, R., Hartmann, F.: Biotechnology Based Building of Clusters in the Region Berlin-Brandenburg. Vortrag auf dem Workshop „Comparing the Development of Biotechnology Clusters" der Akademie für Technikfolgenabschätzung in Baden-Württemberg, Januar 2000.

[6] vgl.: Voß, R., Brandt, M.: Technik für Senioren - ein Zukunftsmarkt für Brandenburger Unternehmen. In: Wissenschaftliche Beiträge der TFH Wildau, Heft 1/2000.

[7] vgl.: Latniak, E. und Rehfeld, D.: Betriebliche Innovation und regionales Umfeld - Erfahrungen in NRW. In: Arbeit Heft 3, Jg. 3 (1994), S. 238-253. Felder, J. et al.: Innovationsverhalten der deutschen Wirtschaft. ZEW-Dokumentation 95-03. Mannheim, 1995. König, H. und Spielkamp, A.: Die Innovationskraft kleiner und mittlerer Unternehmen. ZEW-Dokumentation 95-07. Mannheim, 1995

[8] vgl. Voß, R.: Innovationsmanagement in kleinen mittelständischen Unternehmen - Ansatzpunkte für ein Forschungskonzept mit Beratungsoptionen. Wissenschaftliche Beiträge der TFH Wildau, Heft 1/1996, S. 85-93. Voß, R., Brandt, M., Große, U. und Hartmann, F.: Correlations between innovative behavior of biotech firms and regional development. In press 2001.

[9] vgl. Brandt, M.; Große, U.: Abschlußbericht zum Projekt „Analyse möglicher Wirkungen des Campus Berlin-Buch für eine zukunftsfähige Entwicklung des Bezirks Pankow“, Berlin 1998. Große, U., Brandt, M.: Bedingungen für Beiträge Pankower Unternehmen zu einer nachhaltigen Entwicklung des Bezirks. Berlin 1999

[10] vgl. Hey, Ch., Schleicher-Tappeser, R.: Nachhaltigkeit trotz Globalisierung. Handlungsspielräume auf regionaler, nationaler und europäischer Ebene. Heidelberg 1998, S. 33 
[11] vgl. Biermann, F., Büttner, S., Helm, C. (Hrsg.): Zukunftsfähige Entwicklung. Herausforderungen an Wissenschaft und Politik. Berlin 1997

[12] vgl. Voß, R., Hartmann, F., Große, U. und Brandt, M.: Wissenschaftliche Evaluierung von sechs Modellprojekten regionaler Existenzgründerförderung im Land Brandenburg. Auftraggeber: Wirtschaftsförderungsgesellschaft Elbe-Elster. Berlin/Wildau Oktober 2000

[13] vgl. Weber, J., Schäfer, U., Hoffmann, D., Kehrmann, T.: Technology Assessment. Wiesbaden 1999

[14] vgl. Hartmann, F., Voß, R.: Technikfolgenabschätzung im Bereich Pflanzengentechnik. In: TA-Datenbanknachrichten, Juni 1998. Hartmann, F., Voß, R., Große, U., Brandt, M.: Technikfolgenabschätzung für Innovationslinien auf dem Gebiet der Pflanzengentechnik. In: BioForum 4(2001)

\section{Autoren}

\section{Dr. Martina Brandt}

Technische Fachhochschule Wildau

Forschungsgruppe „Regionale Innovationscluster"

Tel: (0 33 75) 508-512

\section{Dr. Ulla Große}

Technische Fachhochschule Wildau

Forschungsgruppe „Regionale Innovationscluster“

Tel. (0 33 75) 508-512

\section{Dr. Frank Hartmann}

Institut fuir Regionale Innovationsforschung

c/o Technische Fachhochschule Wildau

Tel. (0 33 75) 508-214

E-Mail: Iriinstitut@aol.com

\section{Prof. Dr. Rainer Voß}

Technische Fachhochschule Wildau

Dekan des Fachbereichs Betriebswirtschaft/Wirtschaftsinformatik und Leiter der Forschungsgruppe

„Regionale Innovationscluster “

Tel. (0 33 75) 508-235

E-Mail: rvoss@igw.tfh-wildau.de 